Pacific Journal of Mathematics

ON THE FROBENIUS RECIPROCITY THEOREM FOR 


\section{ON THE FROBENIUS RECIPROCITY THEOREM FOR LOCALLY COMPACT GROUPS}

\section{Calvin C. Moore}

1. Introduction. Let $G$ be a finite group, $K$ a subgroup, and let $M$ and $L$ be finite dimensional representations (over the complex numbers). Then the Frobenius reciprocity theorem (abbreviated as FRT) can be stated in two forms which are readily seen to be equivalent.

(1.) Let $U^{L}$ denote the induced representation of $L$ on $G$ and $(M)_{K}$ the restriction of $M$ to $K$; then there is an isomorphism

$$
\psi: \operatorname{Hom}_{K}\left(L,(M)_{K}\right) \simeq \operatorname{Hom}_{\theta}\left(U^{L}, M\right)
$$

(2.) If $L$ and $M$ are irreducible, then $U^{L}$ contains $M$ exactly as many times as $(M)_{K}$ contains $L$.

The first version is of course formally stronger if one specifies, as is natural, that the isomorphism $\psi$ be functoral.

It is natural to ask how this theorem can be generalized to the context of locally compact groups. Both Mackey and Mautner have defined and discussed the notion of induced representation in this context. Each has formulated a version of the FRT for locally compact groups which generalizes the theorem as stated in form (2) above; [3], [4], [5]. These formulations, however, which use the direct integral decomposition theory, no longer embody the FRT in form (1.) above. It is the purpose of this note to show how one can obtain a version of the FRT which generalizes the theorem in form (1.). The difference between the two versions can conveniently be thought of as a distinction between a global formulation as in (1.) and a local formulation as in (2.).

Concerning our notations, we shall follow a practice throughout and use locally compact to abbreviate locally compact and separable and unitary representation to abbreviate strongly continuous unitary representation on a separable Hilbert space. The notation $\operatorname{Hom}_{\theta}(L, M)$ for representations $L$ and $M$ of $G$ will naturally denote the space of bounded operators $A$ from the space $H(L)$ on which $L$ acts to $H(M)$ so that $A L_{s}=M_{s} A$ for all $s$ in $G$. Concerning induced representations, we adopt the notation of [2].

2. Statement of the theorem. Let $G$ be a locally compact group and $K$ a closed subgroup. We shall assume throughout that $G / K$ (right cosets) possesses a measure $\mu$ invariant under the operation of $G$ by right translations on $G / K$. Such a measure exists if for instance $G$ and

Received February 1, 1961. This work was supported in part by NSF Grant G-14464. 
$K$ are unimodular, [7] $\S 9$; moreover, if $\mu$ exists then it is unique to within a multiplicative constant, [7] $\S 9$. We shall now introduce a notion of induced representation which is a slight modification of that used by Mackey [2].

If $L$ is a unitary representation of $K$ on $H(L)$, define a Banach space $H\left(V^{L}\right)$ as the set of (classes of) weakly measurable functions from $G$ to $H(L)$ so that

$$
\begin{gathered}
f(k x)=L_{k} f(x) \quad \text { for } x \in G, k \in K \\
\int_{G / K}|f(x)| d \mu=|f|<\infty .
\end{gathered}
$$

(The integral has sense since $|f(k x)|=|f(x)|$ for $k \in K$ ). Then we define $\left(\left(V_{s}^{L}\right) f\right) t=f(t s)$ for $f \in H\left(V^{L}\right)$. It is evident that $s \rightarrow V_{s}^{L}$ is a strongly continuous representation of $G$ by isometric operators on $H\left(V^{L}\right)$; moreover, $V^{L}$ is determined up to isometric equivalence by $L$. The representation $V^{L}$ is closely related to the unitary induced representation $U^{L}$ of Mackey. In fact one can define $U^{L}$ using $\mu$ (in place of a finite quasi-invariant measure on $G / K)$; then $H\left(U^{L}\right) \cap H\left(V^{L}\right)$ is dense in both of these spaces and $U_{s}^{L}=V_{s}^{L}$ on this intersection. Thus $U^{L}$ and $V^{L}$ have a dense algebraic subrepresentation in common.

We come now to the statement of the theorem. Let $M$ and $L$ be unitary representations of $G$ and $K$ respectively and $B$ an operator in $\operatorname{Hom}_{K}\left(L,(M)_{K}\right)$. Then we define an operator $\psi(B)$ from $H\left(V^{L}\right)$ to $H(M)$ by the formula

$$
\psi(B) f=\int_{G / K} M_{s}^{*} B f(s) d \mu \quad \text { for } f \in H\left(V^{L}\right) .
$$

The integral has sense since the integrand depends only on the coset of $s: M_{k s}^{*} B f(k s)=M_{s}^{*} M_{k^{-1}} B L_{k} f(s)=M_{s}^{*} M_{k^{-1}} M_{k} B f(s)=M_{s}^{*} B f(s)$ for $k \in K$. The vector valued integral exists in the strong sense in virtue of [1], Theorem 22, p. 117, and Theorem 11, p. 149, since the integrand is clearly weakly measurable, and $\left|M_{s}^{*} B f(s)\right| \leqq|B||f(s)|$ which is an $L_{1}$ function on $G / K$. The last inequality shows that $|\psi(B) f| \leqq|B||f|$ so that $\psi(B)$ is bounded of norm $\leqq|B|$. Finally it is evident that $\psi(B) \in \operatorname{Hom}_{G}\left(V^{L}, M\right)$; for

$$
M_{t} \psi(B) f=\int_{\theta / K} M_{s t^{-1}}^{*} B f(s) d \mu .
$$

Putting $v=s t^{-1}$ and using the invariance of $\mu$ under right translations, we see that this integral becomes

$$
\int_{G / K} M_{v}^{*} B f(v t) d \mu=\psi(B) V_{t}^{L} f
$$


The version of the FRT then reads as follows.

THEOREM. The map $\psi$ is an isomorphism of $\operatorname{Hom}_{K}\left(L,(M)_{K}\right)$ onto $\operatorname{Hom}_{G}\left(V^{L}, M\right)$. Moreover $\psi$ is an isometry between these two Banach spaces in their natural norms.

It is easy to see that a similar theorem with $U^{L}$ in place of $V^{L}$ is false; that $V^{L}$ is the natural representation to consider here is further enforced by the fact that the two spaces of commuting homomorphisms are isomorphic in the strongest possible way, namely isometric.

3. Proof of the theorem. We fix a Borel cross section $S$ of $K$ in $G$ (cf. [2], Lemma 1.1). Then $G / K \approx S$ and we view $\mu$ as a measure on $S$. By restricting functions $f \in H\left(V^{L}\right)$ to $S$, we obtain an isomorphism of $H\left(V^{L}\right)$ with the Lebesgue space $L_{1}(S, \mu, H(L))$. Then the operator $\psi(B)$ is represented as

$$
\psi(B) f=\int_{s} C_{s} f(s) d \mu(s) ; C_{s}=M_{s}^{*} B .
$$

First if $\psi(B)=0$, then for $x \in H(L), g(s) \in L_{1}(S, \mu), g(s) \cdot x \in H\left(V^{L}\right)$, and we have

$$
\int_{S} g(s)\left(C_{s} x, y\right) d \mu(s)=0 \quad \text { for all } y \in H(M) .
$$

Thus $\left(C_{s} x, y\right)=0$ a.e. on $S$. By letting $x$ and $y$ range through countable dense sets $\left\{x_{i}\right\}$ and $\left\{y_{j}\right\}$ in $H(L)$ and $H(M)$ respectively, we can find a common $\mu$ null set $N$ in $S$ so that $\left(C_{s} x_{i}, y_{i}\right)=0$ for all $i, j$ and $s \notin N$. It follows from the continuity of $\left(C_{s} x, y\right)$ in $x$ and $y$ that $\left(C_{s} x, y\right)=0$ if $s \notin N$ for all $x$ and $y$. Now $\left(C_{k^{-1}{ }_{s}} x, y\right)=\left(M_{s}^{*} M_{k} B x, y\right)=\left(M_{s}^{*} B L_{k} x, y\right)=$ $\left(C_{s} L_{k} x, y\right)=0$ for $k \in K, s \notin N$. Then the complement $K \cdot N$ of $K(S-N)$ is a Haar null set in $G\left([2]\right.$, p. $103 \mathrm{ff}$.) and so $\left(M_{s}^{*} B x, y\right)=0$ a.e. on $G$; by continuity, it is zero everywhere on $G$. Therefore $B=0$ and $\psi$ is injective.

Suppose now that $C \in \operatorname{Hom}_{G}\left(V^{L}, M\right)$; we show that $C$ may be represented in the form $\psi(B)$ with $|B|=|C|$. If $x \in H(L)$, define a linear map $C_{x}$ from $L_{1}(S, \mu)$ to $H(M)$ by $C_{x}(g)=C(g x)$. $C_{x}$ is bounded since $\left|C_{x}(g)\right| \leqq|C||g \cdot x|=|C||x \| g|$, and its norm is $\leqq|C||x|$. We may now apply Theorem 10, p. 507 of [1] to conclude the existence of an essentially unique Borel function $D(s, x)$ on $S$ to $H(M)$ so that

$$
C_{x}(f)=\int_{s} f(s) D(s, x) d \mu(s)
$$

and with ess $\sup |D(s, x)| \leqq|C||x|$.

Let $A$ be a subset of $H(M)$ which is countable and dense and a 
vector space over the field $Q(\sqrt{-1})$ of complex numbers of the form $a+i b$ with $a$ and $b$ rational. Since each of the countable number of relations $D(s, \alpha x)+D(s, \beta y)=D(s, \alpha x+\beta y)$ and $|D(s, x)| \leqq|x||C|$ for $x, y \in A$ and $\alpha, \beta \in Q(\sqrt{-1})$ fails to hold only on a $\mu$ null set, it is possible to find a null set $N$ so that they all simultaneously subtend for $s \notin N$. Then the $\operatorname{map} x \rightarrow D(x, s)$ uniquely extends to a bounded linear map $C(s)$ of $H(L)$ to $H(M)$ of norm less than or equal to $|C|$ for $s \notin N$. Now if we define $C(s)=0$ for $s \in N, C(s) x=D(s, x)$ a.e. for each $x$ so that

$$
C_{x}(g)=\int_{s} g(s) C(s) x d \mu(s) .
$$

Finally, if $g \in L_{1}(S, \mu, H(M))$,

$$
C(g)=\int_{s} C(s) g(s) d \mu(s)
$$

since both sides represent bounded linear transformations into $H(M)$ which agree on the dense subspace of $L_{1}(S, \mu, H(M))$ consisting of sums of functions of the form $g x$ for $x \in H(L)$ and $g \in L_{1}(S, \mu)$. Moreover the argument has shown that $C(s)$ is essentially unique and that ess $\sup |C(s)| \leqq|C|$.

In virtue of the Borel isomorphism $G \simeq K \times S$ we may write for $s \in S, t \in G, s \cdot t=k(s, t) l(s, t)$ where $k(s, t) \in K, l(s, t) \in L$. Moreover both $k$ and $l$ are Borel functions on $S \times G$. We shall also make use of the fact that Haar measure on $G$ is equivalent to $\nu \times \mu$ where $\nu$ is Harr measure on $K$ (cf. [2], p. $103 \mathrm{ff}$.). Now let us write $C(s)=M_{s}^{*} B(s)$; then

$$
\begin{aligned}
M_{t-1} C g & =\int_{s} M_{s t}^{*} B(s) g(s) d \mu(s) \\
& =\int_{S}\left(M_{l(s, t)}^{*} M_{k, s, t)}^{*} B(s)\right) g(s) d \mu(s)
\end{aligned}
$$

Also

$$
\left(V_{t^{-1}}^{L} g\right) s=L_{k\left(s, t^{-1}\right)} g\left(l\left(s, t^{-1}\right)\right)
$$

so that

$$
C V_{t^{-1}}^{L} g=\int_{S} M_{s}^{*} B(s) L_{k_{i s, t-1}} g\left(l\left(s, t^{-1}\right)\right) d \mu .
$$

In the second integral let $v=l\left(s, t^{-1}\right)$; since $v t=k\left(s, t^{-1}\right)^{-1} s$, we see that $k\left(s, t^{-1}\right)=k(v, t)^{-1}$, and that $s=l(v, t)$. Making these substitutions, and using the invariance of $\mu$ under the transformation $s \rightarrow l\left(s, t^{-1}\right)$ of $S$ into $S$, we may write the second integral as 


$$
\int\left(M_{l(v, t)}^{*} B(l(v, t)) L_{k(v, t)}^{*}\right) g(v) d \mu .
$$

We now equate the expressions for $M_{t^{-1}} C g$ and $V_{t^{-1}}^{L} C g$ since $C \in \operatorname{Hom}_{G}\left(V^{L}, M\right)$ and use the uniqueness of such integral representations as stated above to conclude that for almost all $s$

$$
M_{l(s, t)}^{*} B(l(s, t)) L_{k(s, t)}^{*}=M_{l(s, t)}^{*} M_{k(s, t)}^{*} B(s) .
$$

Thus for each $t$

$$
B(l(s, t)) L_{k(s, t)}^{*}=M_{k(s, t)}^{*} B(s)
$$

holds for almost all $s$. Now since $k$ and $l$ are Borel functions on $S \times$ $G$, both sides of (*) are Borel functions on $S \times G$ with values in the space of operators from $H(L)$ to $H(M)$ in the sense of the weak Borel structure on the later space.

We may use the Fubini theorem to conclude that for almost all $s$ in $S$ (in particular for some $s_{0}$ ) $(*$ ) holds for almost all $t$ in $G$. Now let us write $t=s_{0}^{-1}(k \cdot v) k \in K, v \in S$; then

$$
k\left(s_{0}, s_{0}^{-1}(k \cdot v)\right)=k, \quad l\left(s_{0}, s_{0}^{-1}(k \cdot v)\right)=v .
$$

Then by use of the equivalence of Haar measure on $G$ with $\nu \times \mu$ as mentioned above and these substitutions,

$$
B(v) L_{k}^{*}=M_{k}^{*} B_{s_{0}}
$$

holds for almost all pairs $(k, v) \in K \times S$. In particular, for some $k_{0}(* *)$ holds for almost all $v$, and we see immediately that $B(v)$ is equal to a constant, let us say $B=M_{k_{0}}^{*} B_{s_{0}} L_{k_{0}}$, for almost all $v$ in $S$. An argument similar to the one just given shows that $B L_{k}^{*}=M_{k}^{*} B$ for almost all $k \in K$, and since $L_{k}$ is weakly continuous in $k$, this holds for all $k \in K$. Then

$$
C g=\int_{S} M_{s}^{*} B g(s) d \mu(s)
$$

for some $B \in \operatorname{Hom}_{K}(L,(M) H)$; $\psi$ is thus surjective. Finally, $|\psi(B)|=$ $|C| \geqq$ ess $\sup |C(s)|=$ ess $\sup |B(s)|=|B|$. Since we have already provd that $|\psi(B)| \leqq|B|, \psi$ is isometric and the proof of the theorem is complete.

REMARK. Formation of the representation $V^{L}$ of $G$ of course removes one from the cozy abode of unitary representations. It would be more natural if $L$ and $V^{L}$ could be taken to be the same generic type. In fact if $L$ is only a representation of $G$ by isometries on a separable Banach space $H(L)$, then representations $V^{L}$ (and $U^{L}$ ) may be defined analogously and these are representations of $G$ by isometries on 
separable Banach spaces. If $M$ is also such a representation, then it is nearly immediate that our theorem remains valid provided only that we assume that $H(M)$ is reflexive or more generally is the dual of (separable) Banach space. Such a condition is necessary to ensure the applicability of the representation theorem (VI 8, 6. ff. [1]) which we have used. The proof remains unchanged except to replace $M_{s}^{*}$ and $L_{k}^{*}$ by $M_{s^{-1}}$ and $L_{k^{-1}}$ respectively; also in case $H(M)$ is the dual of $X$ then the vector valued integrals with values in $H(M)$ which we have used must be taken in the weak- $(*)$ sense.

4. Relation to $U^{L}$. We may use this theorem to obtain information about the unitary induced representation $U^{L}$ [2] in some special cases. We shall assume that $G / K$ admits a finite invariant measure $\mu$. Observe that this condition need not imply that $G / K$ is compact (cf., p. 27 [6]; the space $G / \Gamma$ considered here is not compact). In this case $H\left(U^{L}\right) \cong$ $H\left(V^{L}\right)$, and the embedding map is norm decreasing (assuming as we may that $\mu(G / K)=1)$. Therefore $\operatorname{Hom}_{G}\left(V^{L}, M\right)$ may be viewed as a subspace of $\operatorname{Hom}_{\theta}\left(U^{L}, M\right)$ consisting of these commuting transformations which extend continuously to $H\left(V^{L}\right)$. If in addition the representation $M$ is finite dimensional, it is known, [2], Theorem 8.2 that an exact generalization of form (2) of the FRT holds. This result may be easily deduced with the help of the following.

Proposition. If $G / K$ has a finite invariant measure $\mu$ and $M$ is finite dimensional then $\operatorname{Hom}_{G}\left(V^{L}, M\right)=\operatorname{Hom}_{\theta}\left(U^{L}, M\right)$.

Proof. In virtue of the foregoing remarks, it is enough to show that any $C \in \operatorname{Hom}_{\theta}\left(U^{L}, M\right)$ may be extended continuously to $H\left(V^{L}\right)$. Since $M$ is finite dimensional, this is possible if the linear functional $f \rightarrow(C f, y)$ can be extended continuously to $H\left(V^{L}\right)$ for each $y \in H(M)$. The map $C^{*} \in \operatorname{Hom}_{G}\left(M, U^{L}\right)$ has a finite dimensional range invariant under $U^{L}$. If $f_{1}, \cdots, f_{n}$ is an orthonormal basis, then $U_{t}^{L} f_{i}=\sum_{j=1}^{n} a_{i j}(t) f_{j}$ where the $a_{i j}(t)$ are bounded continuous functions on $G$. Thus for fixed $t$,

$$
f_{i}(s t)=\sum_{j=1}^{n} a_{i j}(t) f_{j}(s)
$$

holds for almost all $s$. Using the Fubini theorem as before, we see that there is some $s_{0}$ so that $(*)$ holds for almost all $t$. It follows immediately that each $f_{i}$ is equal almost everywhere to a bounded continuous function on $G$, and thus $f_{i}$ is essentially bounded. Now if $y \in H(M),(C f, y)=$ $\left(f, C^{*} y\right)=\left(f, \sum_{i=1}^{n} b_{i} f_{i}\right)=\int_{G / H}\left(f(s), \sum_{i=1}^{n} b_{i} f_{i}(s)\right) d \mu$ where $\sum_{i=1}^{n} b_{i} f_{i}$ is essentially bounded. It is then immediate that this formula defines the desired continuous extension of $(C f, y)$ to $H\left(V^{L}\right)$.

Thus under the conditions of the Proposition, there is an isomorphism 
between $\operatorname{Hom}_{K}\left(L,(M)_{K}\right)$ and $\operatorname{Hom}_{G}\left(U^{L}, M\right)$; this result immediately implies the first part of Theorem 8.2 of [2]; namely, if $L$ and $M$ are irreducible, and the hypotheses of the proposition are satisfied, then $U^{L}$ contains $M$ as many times as $(M)_{K}$ contains $L$.

\section{REFERENCES}

1. N. Dunford and J. Schwartz, Linear Operators, Part I. Interscience, New York, 1958.

2. G. W. Mackey, Induced Representations of Locally Compact Groups I. Ann. of Math., 55 (1952), 101-139.

3. - Induced Representations of Locally Compact Groups II. Ann. of Math., (58), (1953), pp. 193-221.

4. F. I. Mautner, A Generalization of the Frobenius Reciprocity Theorem, Proc. Nat. Acad. Sci., U.S.A., $\mathbf{P 7}$ (1951), 431-435.

5. — Induc $c_{\ell}$ Representations, Amer. J. Math., 74 (1952), 737-758.

6. A. Weil, Sur quelques resultats de Siegel. Summa Brasil. Math., (1) (1945-46), 21-39.

7. - L'Integration dans les Groups Topologiques et ses Applications. 2nd Edition, Hermann and Cie., Paris, 1953.

UNIVERSITY OF CHICAGO

ChicAgo, ILLiNoIS 



\section{PACIFIC JOURNAL OF MATHEMATICS}

\section{EDITORS}

Ralph S. Phillips

Stanford University

Stanford, California

M. G. Arsove

University of Washington

Seattle 5, Washington
A. L. Whiteman

University of Southern Californla

Los Angeles 7, California

Lowell J. Paige

University of California

Los Angeles 24, California

\section{ASSOCIATE EDITORS}

E. F. BECKENBACH

D. DERRY

H. L. ROYDEN

E. G. STRAUS

T. M. CHERRY

M. OHTSUKA

E. SPANIER

F. WOLF

\section{SUPPORTING INSTITUTIONS}

UNIVERSITY OF BRITISH COLUMBIA

STANFORD UNIVERSITY

CALIFORNIA INSTITUTE OF TECHNOLOGY

UNIVERSITY OF CALIFORNIA

MONTANA STATE UNIVERSITY

UNIVERSITY OF TOKYO

UNIVERSITY OF UTAH

UNIVERSITY OF NEVADA

NEW MEXICO STATE UNIVERSITY

OREGON STATE UNIVERSITY

UNIVERSITY OF OREGON

OSAKA UNIVERSITY

WASHINGTON STATE UNIVERSITY

UNIVERSITY OF WASHINGTON

UNIVERSITY OF SOUTHERN CALIFORNIA

AMERICAN MATHEMATICAL SOCIETY CALIFORNIA RESEARCH CORPORATION SPACE TECHNOLOGY LABORATORIES NAVAL ORDNANCE TEST STATION 


\section{Pacific Journal of Mathematics}

\section{Vol. 12, No. $1 \quad$ January, 1962}

Jonathan L. Alperin, Groups with finitely many automorphisms $\ldots \ldots \ldots \ldots \ldots \ldots \ldots \ldots$

Martin Arthur Arkowitz, The generalized Whitehead product ................ 7

John D. Baum, Instability and asymptoticity in toplogical dynamics . . . . . . . . . . 25

William Aaron Beyer, Hausdorff dimension of level sets of some Rademacher series .... $\quad 35$

Frank Herbert Brownell, III, A note on Cook's wave-matrix theorem . . . . . . . . . . . . . 47

Gulbank D. Chakerian, An inequality for closed space curves ................. 53

Inge Futtrup Christensen, Some further extensions of a theorem of Marcinkiewicz ....... 59

Charles Vernon Coffman, Linear differential equations on cones in Banach spaces . . . . . 69

Eckford Cohen, Arithmetical notes. III. Certain equally distributed sets of integers . . . . . 77

John Irving Derr and Angus E. Taylor, Operators of meromorphic type with multiple poles

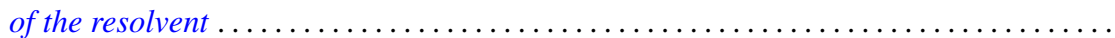

Jacob Feldman, On measurability of stochastic processes in products space .............

Robert S. Freeman, Closed extensions of the Laplace operator determined by a general class of boundary conditions, for unbounded regions ......................

Robert E. Fullerton, Geometric structure of absolute basis systems in a linear topological

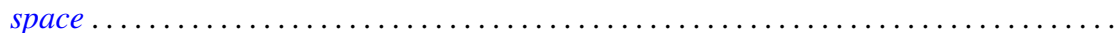

Dieter Gaier, On conformal mapping of nearly circular regions

Andrew Mattei Gleason and Hassler Whitney, The extension of linear functionals defined

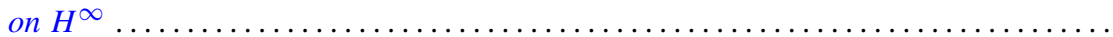

Seymour Goldberg, Closed linear operators and associated continuous linear

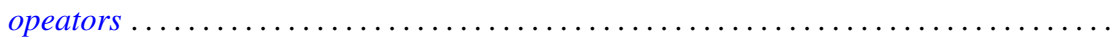

Basil Gordon, Aviezri Siegmund Fraenkel and Ernst Gabor Straus, On the determination of sets by the sets of sums of a certain order

Branko Grünbaum, The dimension of intersections of convex sets. .

Paul Daniel Hill, On the number of pure subgroups

Robert Peter Holten, Generalized Goursat problem . .

Alfred Horn, Eigenvalues of sums of Hermitian matrices ...........

Henry C. Howard, Oscillation and nonoscillation criteria for

$$
y^{\prime \prime}(x)+f(y(x)) p(x)=0
$$

Taqdir Husain, $S$-spaces and the open mapping theorem ...

Richard Eugene Isaac, Markov processes and unique stationary probability measures ...

John Rolfe Isbell, Supercomplete spaces ....................

John Rolfe Isbell, On finite-dimensional uniform spaces. II .........

N. Jacobson, A note on automorphisms of Lie algebras ..............

Antoni A. Kosinski, A theorem on families of acyclic sets and its applications

Marvin David Marcus and H. Minc, The invariance of symmetric functions of singular values...

Ralph David McWilliams, A note on weak sequential convergence.

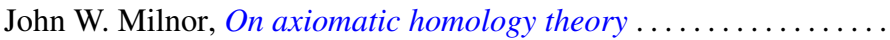

Victor Julius Mizel and Malempati Madhusudana Rao, Nonsymmetric projections in

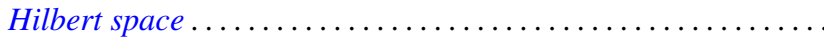

Calvin Cooper Moore, On the Frobenius reciprocity theorem for locally compact

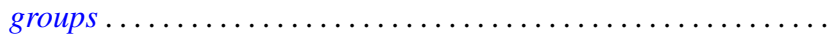

Donald J. Newman, The Gibbs phenomenon for Hausdorff means . 